Switch(config)\#vtp domain screm

Changing VTP 2 domain name from NULL to srcem

Switch(config)\#no vlan 10

\section{DHCP SPOOFING}

As we know that DHCP stands for dynamic host configuration protocol which is used to dynamically assign IP (Internet Protocol) to any device. Additionally, in DHCP spoofing we can easily have configured fake DHCP server to assign the DHCP address to the clients.

\section{DHCP Starvation}

This is DHCP starvation in which any of the attacker consumes all the available IP addresses with change of its MAC (Media Access Control) address. Here we have a new concept of IP address which are issued. Now the server can't issue any new more address for accessing any network.

\section{Defense Mechanism}

Now we can easily observe that here are some attacks which are created on packet tracer affect the layer 2 and 3 are overcome by using the following defense mechanism.

\subsection{Root Attack}

This is a defense mechanism which affects the layer 2 and layer 3 using packet tracer. Now we can easily enable root guard. In addition to this we can also the VLAN group.

\subsection{DTP Attack}

For preventing DTP attack port security feature is used. If another MAC address device use the port then port of the switch automatically becomes off. We can also prevent the DTP attack by configuring access mode and disable dynamic trunking protocol.

\subsection{Routing Protocol Attack}

If inner port of the router is configured as passive port then no updates will exchanged with the attacker.

\subsection{DHCP Starvation and Spoofing attack}

Nowadays we have an additional feature of port security. In addition to this we can also shut down the unused ports. Now we can say that by using the above two methods we can easily get from the problem. This is called DHCP starvation and spoofing attack. Also time limit is also configured to the DHCP server for assigning the IP address.

\section{VIII. results}

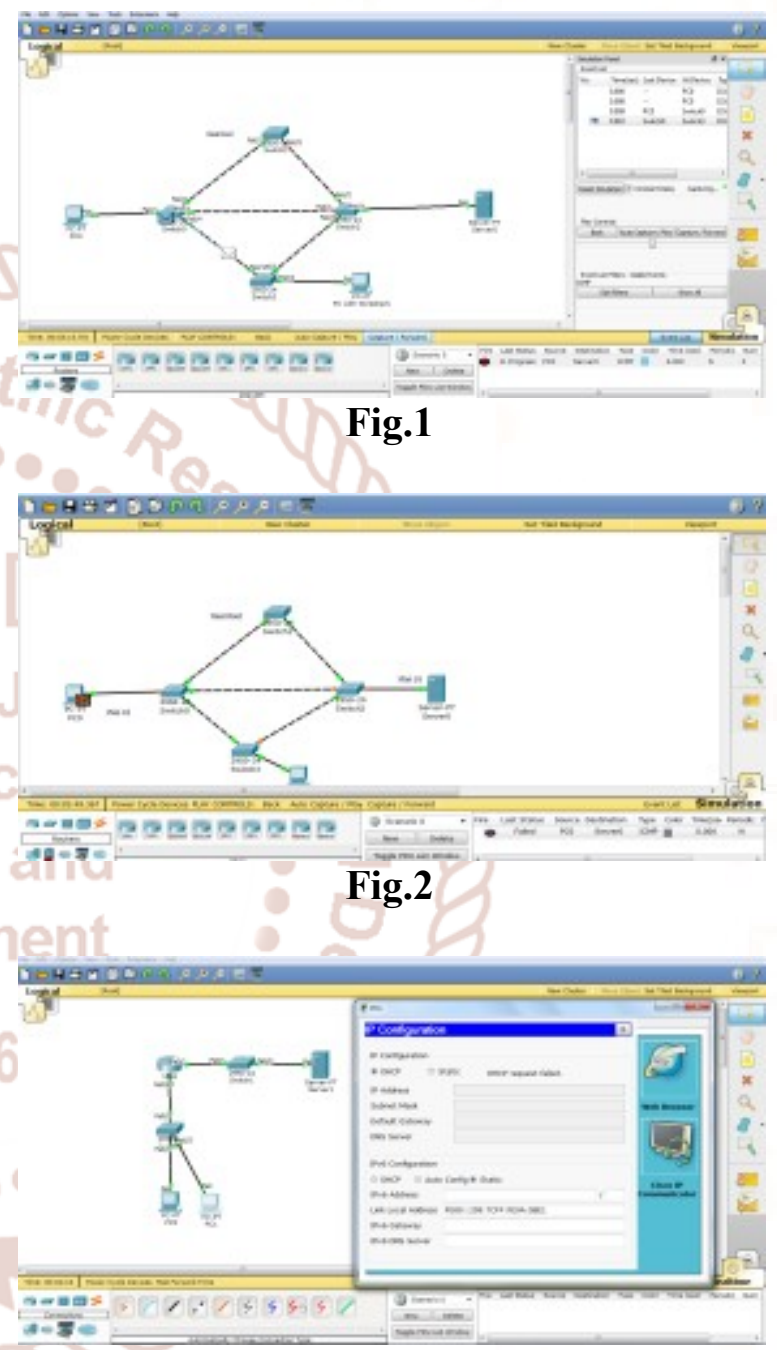

Fig.3

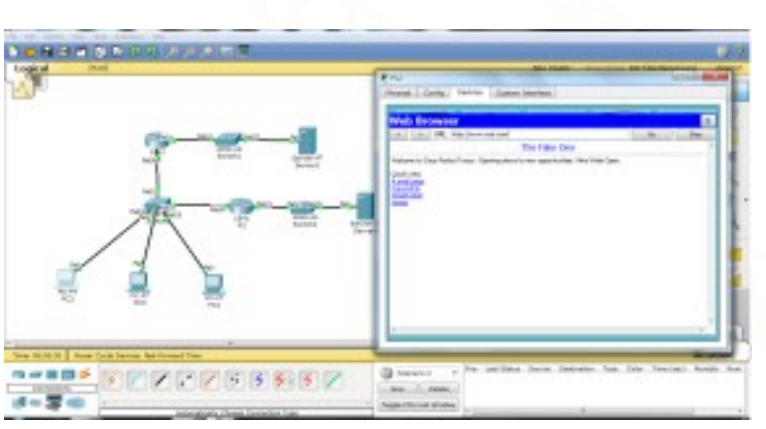

Fig.4 


\section{Conclusions}

After reading this technical paper we can easily predict that the two layers including data link layer as well as network layer both are vulnerable to attacks including spoofing attack, DHCP starvation and DTP attack also. Now we can say that port security can be considered as one of the trustworthy method. In addition to this we can also say that root attack can also be overcome by enabling root guard and BPDU guard. Additionally we can also consider the concept of AD (Administrative Distance) for selecting the optimum path.

In this thesis, attacks are shown and demonstrated using a simulator called "PACKET TRACER".

\section{References}

1) "Exploiting DHCP Server-side IP Address Conflict Detection: A DHCP Starvation Attack',Nikhil Tripathi, Neminath Hubballi Conference Paper · December 2015.

2) "Tracking Low Grade Attack Using Cisco Packet Tracer Netflow" Manish khule1, Megha Singh2, International Journal of Emerging Technology and Advanced Engineering, ISSN 2250-2459, Volume 5, Issue 1, January 2015.

3) "Increasing Network Efficiency By Preventing Attacks At Access Layer", G.Narasimha, M. Jithender Reddy, International Journal of Research in Engineering and Technology Volume: 03 Special Issue: 05 | May-2014, eISSN: 2319-1163| pISSN: 2321-7308.

4) "A Review of types of Security Attacks and Malicious Software in Network Security" Inam Mohammad and Rashi Pandey, International Journal of Advanced Research in Computer Science and Software EngineeringVol.4, Issue 5,May-2014 ISSN: 2277 128X.

5) "Investigation of DHCP Packets using Wireshark", Mohsin khan, Saleh Alshomrani, Shahzad Qamar, International Journal of Computer Applications (0975 - 8887) Volume 63- No.4, February 2013.

6) "Secure ARP and Secure DHCP Protocols to Mitigate Security Attacks", Biju Issac International Journal of Network Security, Vol.8, No.2, PP.107-118, Mar. 2009.

7) "Tools for Attacking Layer 2 Network Infrastructure" Kai-Hau Yeung, Dereck Fung, and Kin-Yeung Wong, International Multi Conference of Engineers and Computer Scientists 2008 Vol II IMECS 2008, 19-21 March, 2008, Hong Kong.

8) www.cisco.com 